\title{
Adaptation of the Higher Education in Engineering to the Advanced Manufacturing Technologies
}

\author{
Olivier Bonnaud ${ }^{1,2,}$, Ahmad Bsiesy ${ }^{3,4}$ \\ ${ }^{1}$ Department of Sensors and Microelectronics, IETR, University of Rennes 1, 35042 Rennes, France \\ ${ }^{2}$ National Coordination for Education in Microelectronics, GIP-CNFM, 38016 Grenoble, France \\ ${ }^{3}$ University Grenoble Alpes, CNRS, LTM, F-38000 Grenoble, France \\ ${ }^{4}$ CIME Nanotech, University Grenoble-Alpes, 38016 Grenoble, France \\ Received 25 April 2019; received in revised form 07 July 2019; accepted 02 November 2019 \\ DOI: https://doi.org/10.46604/aiti.2020.4144
}

\begin{abstract}
The 21st century will be the era of the fourth industrial revolution with the progressive introduction of the digital society, with smart/connected objects, smart factories that are driven by robotics, the Internet of Things (IoT) and artificial intelligence. Manufacturing is expected to be performed by smart factories, i.e. the industry 4.0, which is the outcome of steady development of information technology associated with new objects and systems that can automatically supervise and fulfil manufacturing tasks. The industry 4.0 concept relies largely on the ability to design and manufacture smart and connected devices that are based on microelectronics technology. This evolution requires highly-skilled technicians, engineers and $\mathrm{PhDs}$, all of them well prepared for research, development and manufacturing. Their training, which combines knowledge and the associated compulsory know-how, is becoming the main challenge for the academic world. The curricula must therefore include the basic knowledge and associated know-how training in all the specialties of the field. The software and hardware tools used in microelectronics education are becoming highly complex and expensive that the most viable solution for practical training is to share technical facilities and human resources. This strategy has been adopted by the French microelectronics education network, which includes twelve universities and two industrial unions. By sharing human resources and technical facilities, the CNFM network was able to minimize the costs and to train future graduates on up-to-date tools similar to those used in companies. This paper aims to show how the strategy adopted by the French network can help meeting the needs of the future industry 4.0. Several examples of innovative activities developed in this strategy will be given.
\end{abstract}

Keywords: industry 4.0, microelectronics, engineering education, network structuring, multidisciplinarity

\section{Introduction}

The evolution towards digital societies implies a revolution in the industry that relies largely on the tremendous growth of microelectronics, which is at the heart of the Internet of Things (IoT) [1-2]. Indeed, the rapid development of microelectronics [3] has made it possible to achieve fast computing, high-speed data processing and communications, and also more sensitive detection and more effective actuation [4]. Today, all these functions are integrated in the connected objects that apply to all major societal activities [5], from communications to health, safety, transport, energy [6-7], as well as production in the new industrial scheme [8]. In fact, modern industry takes advantage of intelligent robots [9-10] that are well adapted to the

* Corresponding author. E-mail address: olivier.bonnaud@univ-rennes1.fr 
production environment and have a core based on connected objects. As a result, industry is increasingly dependent on IoT, which is necessary to govern the design of new products, their production, as well as to control the flow of raw materials and manufactured objects. All these production and management functions are part of what is known as smart factory industry 4.0 [8]. In this field, engineers, technicians and $\mathrm{PhD}$ grade holders must be integrated into this design and manufacturing chain. The skills of these actors must combine the knowledge and practical know-how or skills that are essential when manufacturing a real object integrated into its environment and with a well-defined mission profile [11]. Electronic hardware platforms (microprocessors, microcontrollers, sensors, actuators, etc.) are at the heart of the smart factory and they are provided by microelectronics industry [12]. Higher education programs in engineering must therefore contain the basic knowledge and associated know-how in the following specialties: analog and digital electronics, signal processing, sensors and actuators, on-board electronics, energy harvesting systems, communications, transmission protocols and human-machine communication systems (vision, sound, touch, etc.) [6]. These specialties must meet the future needs of a connected society: low power, high speed, reproducibility, quality, safety, reliability, and low cost. A transformation of higher education is a major challenge to meet the needs of companies and research centers with a permanent behavior oriented towards innovation. This paper addresses these different aspects.

\section{Microelectronics and IoT}

As mentioned above, microelectronics and its evolution towards nanotechnologies are the driving force behind the evolution towards the digital society and future industry 4.0. Over the past sixty years, the integration of elementary devices into integrated circuits, mainly transistors, has grown exponentially, primarily due to the decrease in the lateral dimension of elementary devices. This evolution followed the famous Moore's Law [3]. Nowadays, the minimum dimension entered within the nanometre scale, which is why we are now talking about nanotechnologies. On the one hand, the reduction in size was governed by a better control of the stages of the manufacturing process gradually involving the self-assembly of nano-objects and thin films. On the other hand, the size has also decreased due to the increase in the computing capacity that led to the improvement in the design of increasingly complex circuits. The consequence of this unprecedented evolution is that the lateral dimensions of the devices are currently approaching the inter-atomic distance. Thus, the decrease in the size of the elementary transistors and thus the increase in the integration density of the transistors reach a physical limit. However, since the early 2000s, the introduction of the third dimension has provided a new way to further increase integration density. Indeed, this approach is based on stacking of elementary devices and circuits on the surface of the previous circuits. This is carried out by involving new deposition techniques of thin films, and the technique of stacking of the circuits themselves based on the thinning of the substrates followed by a sticking process [13-14]. Fig. 1 illustrates these two developments. The number of elementary devices in the most advanced electronic systems can count up to several billions [3].

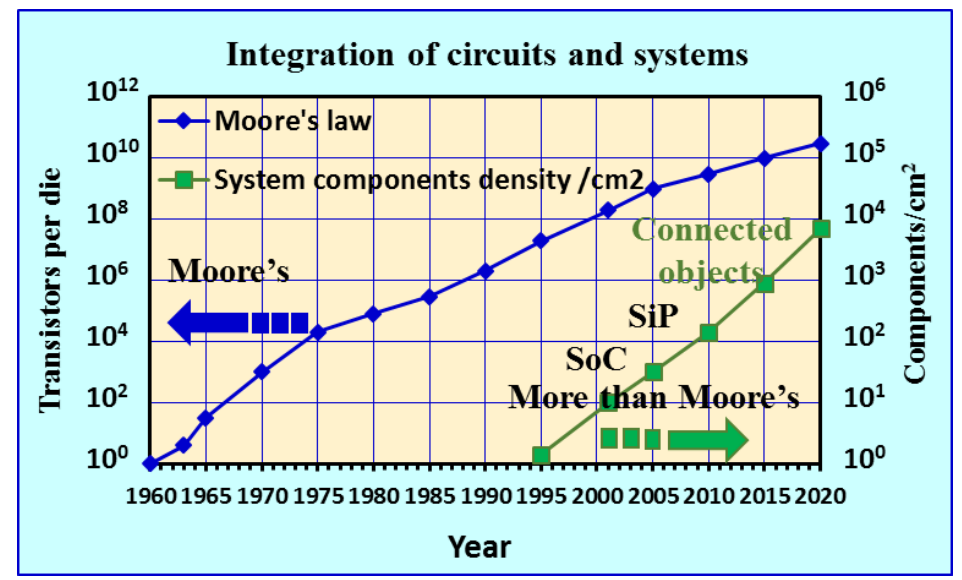

Fig. 1 Moore's Law [3] and "More than Moore" evolution [13]. Systems on chip (SoC) and systems in package (SiP) open the way for connected objects [14] 
Thanks to this integration scheme, many electronic functions have been developed and by combining several technological processes, the extension to multi-physical systems has become possible. For example, micro-electro-mechanical systems (MEMS) technology opened the way to create many types of sensors and actuators. The possibility to gather several functions allows the creation of systems on chips ( $\mathrm{SoC})$ and systems in package ( $\mathrm{SiP}$ ) which are the cornerstone of new connected objects. Indeed, the connected objects are a combination of several functions that are summarized in Fig. 2 [5-6]. They may contain sensors, actuators, signal processing modules, analog/digital and digital/analog converters, energy harvesting cells, and communication modules. Depending on the application domain, sensors and actuators are multidisciplinary [15]. With these new systems, the fields of application can cover all societal needs such as communications, health, environment, energy, transport, agriculture, and the new industry 4.0, which is covered in this document and that requires advanced manufacturing technologies.

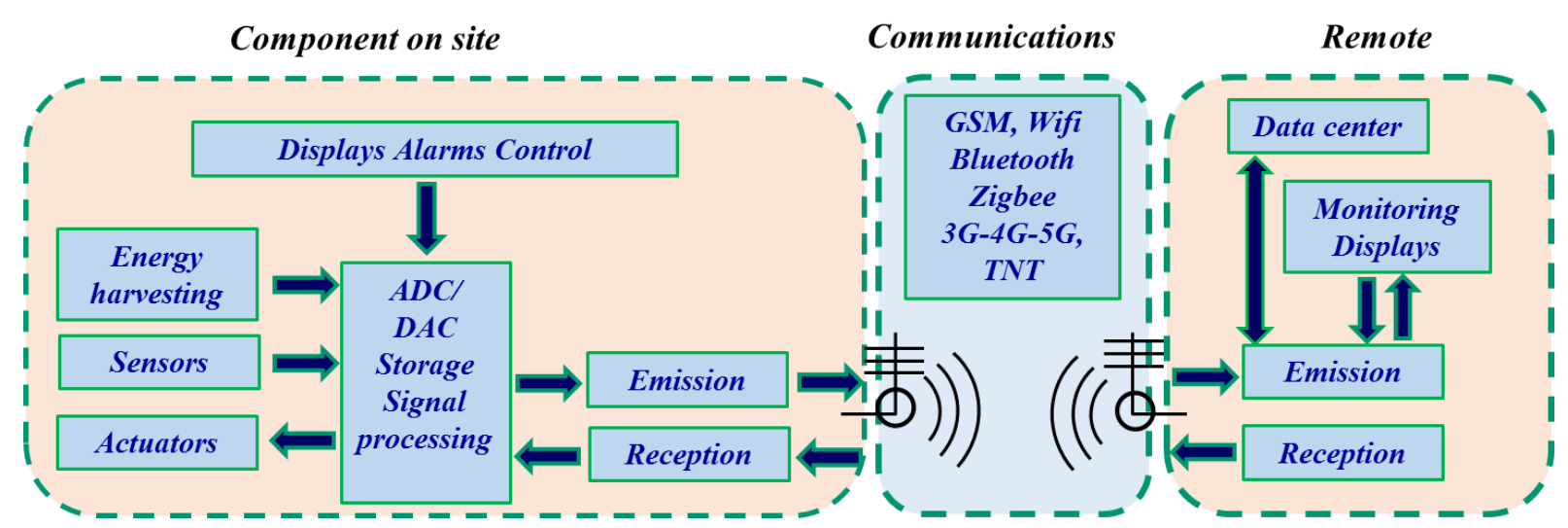

Fig. 2 Simplified architecture of a connected object with its main functions [6]

To summarize, the microelectronics and the associated new connected objects, allow the complexity of the architecture and the systems, increasingly involve multidisciplinary approach, and can be applied to large spectrum of societal needs in the so-called Internet of Everything or IoE [16-17]. The human resources associated with this field, i.e. the engineers, technicians, and operators working in the manufacturing have to acquire skills corresponding to knowledge and know-how of the field, associated with capabilities of team working.

\section{Advanced Manufacturing Technologies and Constraints}

Advanced manufacturing technologies should involve many objects, including robots and connected environments. This environment must be secure and reliable in terms of physical objects, intellectual property, data protection and monitoring. For designers and manufacturers, this means a lot of works are dedicated to the safety and reliability of objects and microelectronic circuits, as well as to data transmission and the reduction of possible interferences in the signals. All these points put high challenges on microelectronic devices, circuits and systems.

Another aspect is less visible but of ever-increasing importance. It is the energy consumption of all these objects within the IoT framework. Fig. 3 shows the expected evolution of IoT energy consumption over the next 20 years. Growth is exponential and IoT electrical power consumption is expected to reach 15,000 GW in 2040, which is the global energy consumption from all sources (nuclear, oil, gas and renewable energy) by 2018 [18], while the global average power consumption should reach about $125,000 \mathrm{GW}$ [19-20]. It is clear that the planet will not be able to reach this level of production and the reasonable solution is to significantly reduce the consumption of each connected object by a factor of more than 10 and probably close to 100 after 2040 .

The solutions to this issue require deep changes in current microelectronics field. It is still necessary to reduce the consumption of each elementary device thanks to nanotechnology development, optimize the number of components in the 
circuits, create new architectures that sequentially activate the useful zones by putting all the others on standby, i.e. to move from synchronous architectures to asynchronous ones in order to minimize the consumption of the clocks, combine different technologies (ULSI, thin film technologies, organic technologies) and improve the transmission and reception efficiency in signal transmissions, to mention only the most obvious ones.

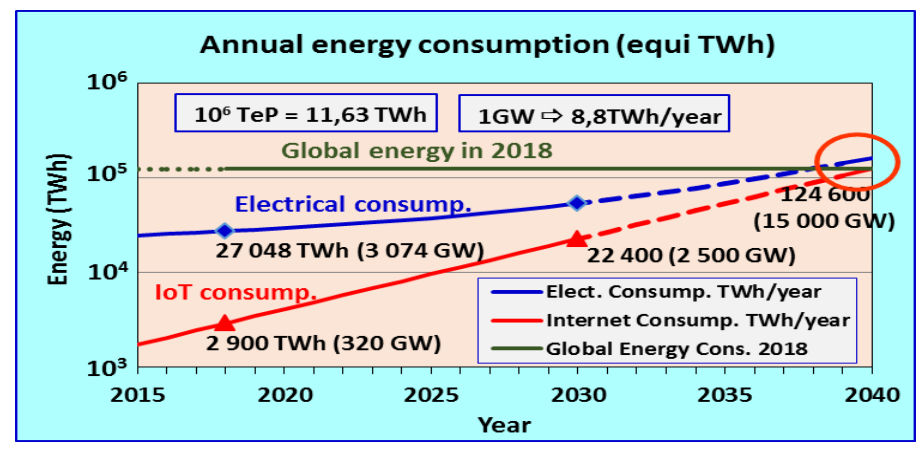

Fig. 3 Projected annual energy consumption over the next 20 years, equivalent to the world average annual capacity in 2018, all energy sources cumulated (After [20])

Creating electronic circuits in heterogeneous technologies and multidisciplinary applications, improving safety, controlling energy consumption are the challenges that future technicians, engineers and PhDs will have to face. They must therefore be prepared through initial and lifelong training adapted to this evolution. University training in engineering must therefore meet these needs. The French Network for Education in Microelectronics and Nanotechnology (CNFM) [21], created in the early 1980s and moving from microelectronics to nanotechnology in the 2000s [22], has adopted a strategy in this direction.

\section{French Higher Education Network in Microelectronics}

\subsection{The CNFM French network}

Higher education in microelectronics in France is coordinated by a 35-years old national network, the CNFM, funded by the Ministry of Higher Education with an official structure called "Groupement d'Intérêt Public" (GIP) [21-22]. This network was created to share education facilities (platforms) containing expensive micro and nano-fabrication equipment and tools, that require high operating costs. These shared open facilities allow offering practical training to all academic institutions in France that become users of the platforms for training their students in know-how. Foreign academic institutions are also users of the platforms in the frame of cooperation agreements.

This network is composed of twelve academic members who are respectively in charge of the twelve common centers spread over the French territory as shown in Fig. 4, each center being common to several academic institutions located in the same geographic area. The twelve CNFM common centers (red labels) manage platforms i.e. technical facilities for practical hands-on training. Among the eighty technical platforms, 7 cleanrooms are mainly dedicated to Education (red stars). National services for CAD (Computer-Aided-Design) tools provided by the Montpellier CNFM common center are dedicated to all the national community and foreign partners of the network. Two industrial unions and especially the most representative association ACSIEL Alliance Electronics consortium [23] are also the members of the CNFM body. They represent more than 150 industrial sites (orange and green circles). The presence of industrial partners ensures strong links between academic training and industrial needs. Indeed, industrial partners provide the CNFM network with the necessary valuable advice on the nature of skills and competencies needed by the work market. Hereafter, we will present what we think to be the main characteristics of a good education approach able to ensure i) a high quality of the content, ii) a know-how on updated and industrial tools, iii) an international recognition of the diploma, iv) and sustainability meeting the needs of advanced manufacturing technologies. 


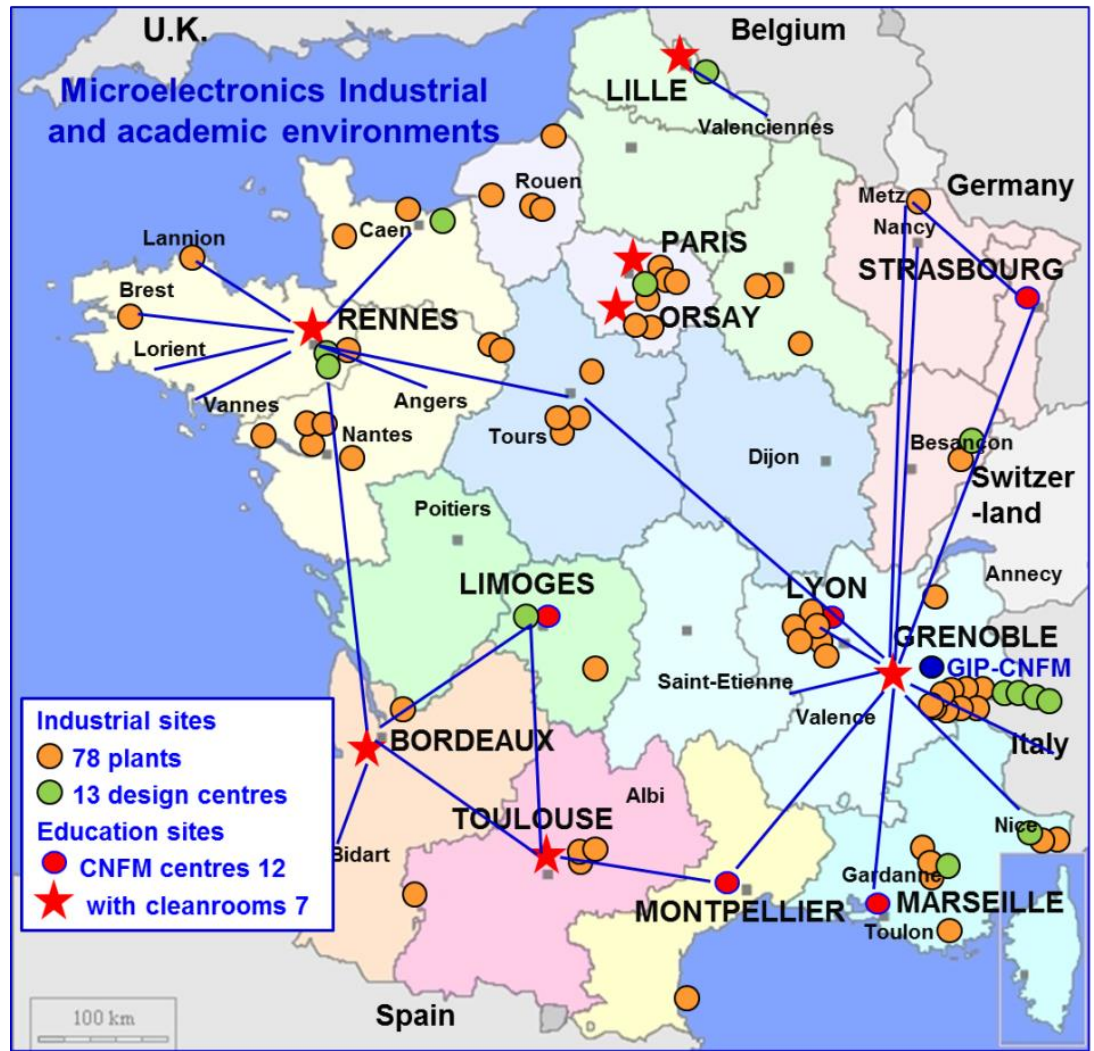

Fig. 4 Map of the French academic and industrial activities in microelectronics [21]

The main company sites are identified by orange circles. The twelve common centres of the CNFM (red circles and red stars) manage practical training platforms and are located throughout the metropolitan area. For their practice in cleanroom, students travel from their institution to closest CNFM centres (blue lines).

\subsection{The missions and the strategy of the CNFM network}

As previously mentioned, the very essence of the GIP-CNFM network is to share technical platforms, which include technological processes, CAD, characterizations, and test, because of their very high equipment and operating costs. In addition, sharing pedagogical approaches between educators, and applying good practices towards students trained in initial training or even in LifeLong Learning (LLL) is also a main educational target. Collaboration within the network exists in several forms, via:

- the sharing and common use of technological and design platforms,

- the collaborative work in the context of national, or international, multi-year projects,

- three-day educational workshops and brainstorming seminars involving teachers, researchers and technical collaborators, as well as industry representatives.

These joint activities allow the exchange of knowledge and practices in order to produce and disseminate knowledge and know-how to the entire academic community [24] as well as to foster an innovative approach that is mandatory for advanced manufacturing technologies.

\subsection{CNFM strategy with industry}

The main point of the GIP-CNFM's strategy is to pool technology, design, characterisation and testing platforms in the broad field of electronics, microelectronics and nanotechnologies. This network provides a permanent link between the academic world and the industrial world that can reflect its needs in the short, medium and long term. The technological 
platforms are accessible to all students, whatever their level from undergraduate to post-doctoral students, which allows them to acquire essential know-how in addition to the theoretical knowledge acquired either in traditional form (courses) or in the form of online tools, such as MOOCs [25-26]. Since electronics and microelectronics are at the heart of the majority of innovative connected objects, the training strategy aims to provide skills and know-how in these fields [27] but also in the fields of application such as communication, health, environment, transport, etc. [15]. The existence of multidisciplinary platforms open to initial and lifelong learning must make it possible to meet the needs of industry in terms of the quality and relevance of curricula content.

The close link within the CNFM network between academia and industry makes it possible to jointly define learners' needs, build curricula adapted to the objectives and ensure learning that includes both the knowledge and know-how required for a successful 21 st century industry [27].

\subsection{Collaboration within the network and international extension}

Collaboration within the network exists in several forms, on the one hand through the sharing and common use of technological and design platforms, and on the other hand through collaborative work in the context of national, or international, multi-year projects and through the organisation of steering committees, national educational workshops and reflection seminars, particularly within the framework of the network's Orientation Council. These joint activities allow for the exchange of knowledge and practices in order to produce and disseminate knowledge and know-how to the entire academic community.

This good practice of networking is not so familiar within the French academic world and serves as an example to other fields both in France and also abroad. The use of platforms shared by several academic institutions allows users from different backgrounds and belonging to different institutions to meet and collaborate while acquiring know-how that is essential for all.

\section{Some Answers to the Needs of Companies Involved in Manufacturing}

With the objective of best meeting the needs of manufacturing industry, several parameters and qualities must be taken into account following several brainstorming meetings during 2018 and 2019. Their objective is to adapt the training of technicians, engineers and PhDs, who will have to get along well with companies in industry 4.0. In the qualities required, we find constants, new approaches, and skills.

\subsection{Adaptation of the content}

The main aim is to cover the entire design and manufacturing chain of the connected objects that will be at the heart of 21st century industry. The programmes should therefore contain the basic knowledge and associated know-how in the following specialities: analog and digital electronics, signal processing, sensors and actuators, embedded electronics, energy harvesting systems, communications, transmission protocols and human-machine communication systems (vision, sound, touch, etc.). Thanks to the annual funding provided by the CNFM to innovative education projects in the frame of the FINMINA project [28], the platforms are permanently up-dated with new practice in accordance with the evolution of the techniques and the fields of application [29]. These specialities must meet the future requirements of a connected society, namely: low power, low consumption, speed, reliability, low cost. In fact, the 80 platforms cover the entire microelectronics spectrum.

\subsection{Learning environment}

The objective of the existing network is to provide a learning environment that allows the trainees to be immersed in a technological environment that prepares them for the industrial environment of the 21 st century and more particularly for 
industry 4.0. Indeed, the current centres include seven cleanrooms that condition users to the requirements of advanced technologies, namely, cleanness concept, adapted clothing, handling with dedicated tools or robots. Indeed, the current centres include seven clean rooms that show the users the requirements of advanced technologies: cleanliness concept, adapted clothing, handling with dedicated tools and robots. They allow the use of high chemical purity products to minimize the contamination of integrated electronic devices, and the use of complex equipment that allows the creation of thin layers with thickness control at the atomic scale. The platforms associated with these cleanrooms allow a multidisciplinary orientation by the realization of MEMS (Micro-Electrical-Mechanical-Systems), BIOMEMS (Biological MEMS), OMEMS (Optical MEMS), intended for sensors or actuators of connected objects [4]. Computer-aided design of electronic systems uses industrial software to acquire concepts that capture high-level abstract models. These electronic systems are usually described by the means of advanced computer languages (such as VHDL) that design the functionalities, and non-functional specifications such as electrical consumption, safety, reliability and performance. Due to the complexity of integrated systems, design requires the juxtaposition of many skills and know-how, which implies teamwork and therefore collective problem solving. Team project training must meet these requirements and becomes an essential form of the pedagogical approach.

Within the network, all colleagues apply these principles in the educational programmes of their partner institutions, which automatically implement an experimental approach.

\subsection{Delivery mechanisms}

As already mentioned above, the first objective of the network is to provide the corresponding knowledge and know-how. Given the organisation of the network, teachers and learners interact face-to-face on concrete issues. Indeed, any practical activity is closely supervised because of the complexity, impact and dangerousness of the equipment used. This strong support in practical activities can be compensated by more autonomy in acquiring basic knowledge using online tools such as MOOCs [26, 30], serious games [31] and flipped classrooms [32]. These tools can also be adapted to prepare practical activities (practical training, supervised projects, etc.) in order to optimize the presence on the platforms and minimize their cost. The introduction of virtual and augmented reality or artificial intelligence can be considered as part of a constructive cooperation between the existing CNFM network and other networks with these competences.

\subsection{Assessment}

Since practical activities in a high-tech environment are automatically supervised by a tutor or teacher, the evaluation must first be done by the supervisor. However, as these activities are generally carried out in groups, it is quite possible to exploit the group dynamic and its self-assessment, which can be included in experience reports drawn up at the end of their practical training on platforms.

\subsection{Recognition}

The official CNFM training network is made up of service units (or CNFM poles), which are not a priori authorised to deliver a diploma. Diplomas, certificates or titles are awarded by the academic institutions to which the student users are attached. However, as part of lifelong learning, certificates may be issued specifying the objectives, content and quality of the work carried out on the CNFM platforms. It is expected that the network will become a certification body specifically designed for the training of employees of companies using the network's resources.

\subsection{Quality}

The content of the essentially practical activities are established in connection with academic training on the one hand and with companies for their employees on the other. This approach ensures content quality. At the end of practical training, 
particularly for continuing training (LLL), questionnaires are drawn up to check that the actual training is in line with the objectives defined in advance and that the trainees' perception is correct. A debriefing at the end of the training session should also allow trainers to justify certain approaches that would not have been perceived in the same way by users.

\subsection{Sustainability of the education network}

The CNFM network's strategy, which makes it possible to pool resources and maintain good matching with the needs of the socio-economic world, can only be achieved by keeping platform equipment and study topics at the highest level. The field of microelectronics has been evolving very rapidly for decades and training must also keep the pace.

Thus, this policy requires financial support to update the hardware and software that academic bodies currently have difficulty in assuming despite the pooling organised by the network, which however makes it possible to limit the cost. It is necessary to consider at national and European levels, that the training needs of companies are specifically supported within the framework of industrial sectors with dedicated funding from both public bodies and companies.

\section{Adaptation of the Technical Content by the CNFM Network}

\subsection{New technical challenges}

As a result of the analysis of the context, the technical challenges that the future engineers and doctors will have to face appear at different levels in order to improve the electrical behaviour and the electrical energy consumption of the future connected objects included in robots or cobots, and involved in the advanced manufacturing. Their interventions should occur at the level of the elementary devices, of electronic functions and circuits and at the level of systems, as follows:

- Continuation of the miniaturization of elementary devices, minimizing consumption both in the on-state (Ron) and off-states (leakage),

- Reduction of switching and static losses of power components,

- Introduction of the third dimension to improve circuit integration in minimizing interconnection losses and improving reliability,

- New circuit architectures able to control active and stand-by zones,

- New circuit concepts allowing the generalization of asynchronous control of all elementary electronic function, minimizing the synchronous power supply by the clocks of all transistors,

- IMPLEMENTATION of low temperature and large area technologies,

- Development of low power sensors and actuators,

- Optimization of communication devices and protocols to limit the occupation of frequency bands and the data flow.

It is clear that all these topics will need capabilities of team working and some multidisciplinary skills for the actors [34]. The choice of the practice of the network thanks to the annual calls organized by the network council is deliberately oriented towards these technical challenges. In the following we give several examples adapted to this strategy.

\subsection{Example of innovative practice provided by the CNFM centres}

Several examples of realization by students in the CNFM centres during the last years are given. The scope is wide and we highlight only a few images that are representative of the orientation towards advanced manufacturing technologies. Fig. 5 shows the designed and fabricated objects and mentions the corresponding CNFM centre of realization. 


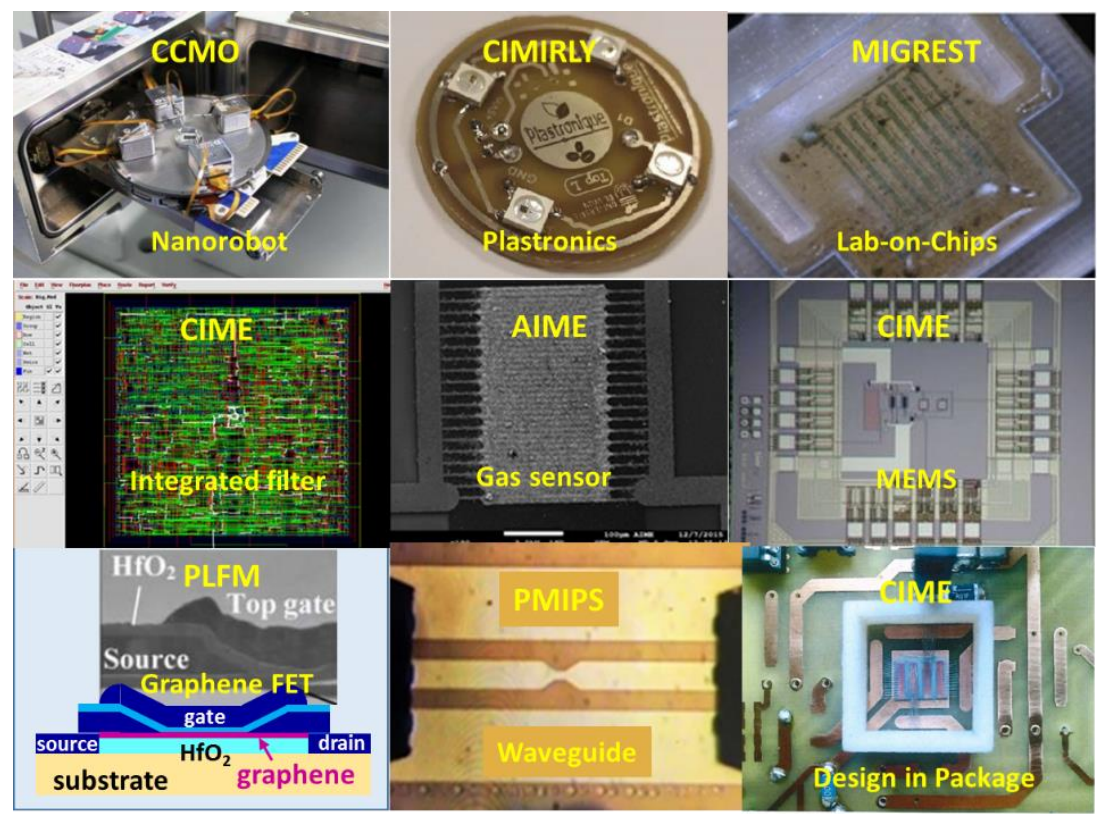

Fig. 5 Examples of objects, designed, fabricated and characterized in the microelectronics centres

These examples show the complexity of the practice training on the different platforms, the multidisciplinarity of the realizations which are adapted to the local context of the microelectronic centres: computer-aided-design of new architectures for low consumption circuits [33], new sensors and micro-electrical-mechanical systems (MEMS), new nano-devices involving nanowire-based transistors and two-dimensional graphene-based transistors, radio-frequency waveguides for transceivers, electronics on plastics (Plastronics), and lab-on-chip. Depending on the complexity of the realization, the students can acquire the knowledge in less than one week in general initial formation, or during several weeks in the frame of projects that are more specialized. The students are using the design tools and the clean-room and characterization facilities of the different centres and of the national services for CAD software [34].

\subsection{Example of innovative practice provided by the centers}

Since 2011-2012, more than 100 innovative projects have been launched by the CNFM centres. The users, mostly master's and engineering degree students, have experience in practical training and more particularly on innovative subjects. Fig. 6 shows the evolution of the network activities over the past seven years.

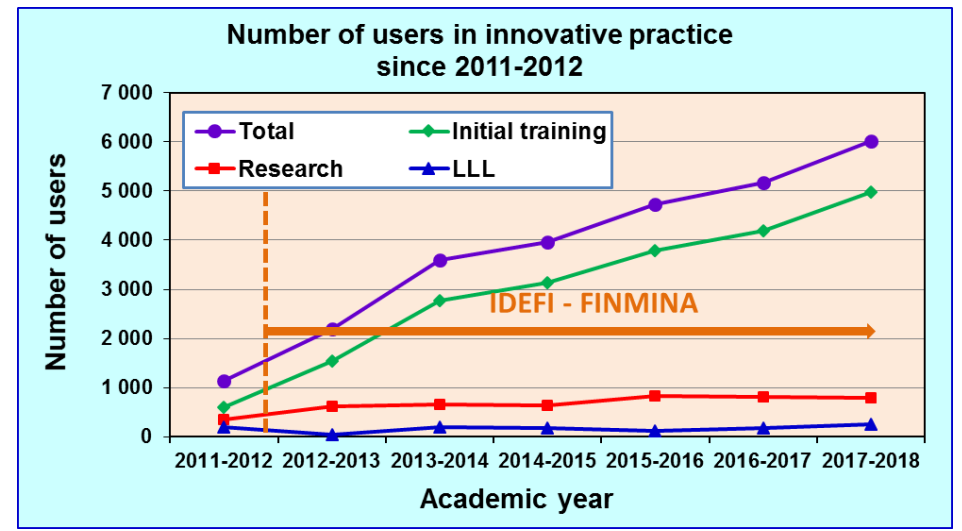

Fig. 6 Innovative practice on the platforms of the CNFM network over the past six academic years

Thanks to the national FINMINA programme [28], more than 6,000 students have acquired experience and know-how on new technological or design tools that meet the new needs of industrial and societal applications. About 1,000 are users of the platforms during their $\mathrm{PhD}$, and about 300, mainly employees of companies, are in lifelong learning in a thematic reconversion cycle. 


\section{Conclusions}

The emergence of advanced manufacturing technologies in industry 4.0 requires an increasing adaptation of the training of engineers and technicians in the field of microelectronics to ensure that they acquire the skills and know-how necessary for the ever-changing IoT technologies. The adaptation of training, which must meet quality and efficiency criteria, to the acquisition of skills and know-how is essential to meet economic, industrial and social needs [35]. The French microelectronics and technology training network, with the help of its twelve common platforms with a national mission, enables innovative practical activities to be set up using high-performance tools. This approach, considered original by the international academic community and highly appreciated by French industry, can serve as an example for other foreign countries.

\section{Acknowledgment}

The authors want to thank all the members of the French GIP-CNFM network for they contribution to many innovative realizations. This work is financially supported by French Higher Education Ministry and by IDEFI-FINMINA program (ANR-11-IDFI-0017). A special thanks to L. Chagoya-Garzon, secretary of GIP-CNFM for her fruitful advice for the proof reading of this paper.

\section{Conflicts of Interest}

The authors declare no conflict of interest.

\section{References}

[1] H. Chaouchi, “The Internet of Things: Connecting Objects,” ISTE Ltd, John Wiley \& Sons, May 2010.

[2] M. Burgess, "What is the Internet of Things?," WIRED Magazine, https://www.wired.co.uk/article/internet-of-things-what-is-explained-iot, February 16, 2018.

[3] G. E. Moore, “Cramming more components onto integrated circuits,” Electronics Magazine, vol. 38, no. 8, pp. 114-117, 1965.

[4] V. Sharma and R. Tiwari, “A review paper on "IOT” \& Its Smart Applications," International Journal of Science, Engineering and Technology Research, vol. 5, no. 2, February 2016.

[5] A. Schütze, N. Helwig, and T. Schneider,"Sensors 4.0-smart sensors and measurement technology enable Industry 4.0," Journal of Sensors and Sensor Systems, vol. 7, no. 1, pp. 359-371, May 2018.

[6] O. Bonnaud, "New Approach for Sensors and Connecting Objects Involving Microelectronic Multidisciplinarity for a Wide Spectrum of Applications," International Journal of Plasma Environmental Science and Technology, vol. 10, no. 2, pp. 115-120, December 2016.

[7] G. Matheron, "Microelectronics evolution, Keynote, European, Microelectronics Summit," Paris, France, November 2014.

[8] B. P. Santos, F. C. Santos, and T. M. Lima, "Industry 4.0: an overview," Proceedings of the World Congress on Engineering, vol. 2, 2018.

[9] M. Peshkin and J. E. Colgate, “Cobots,” Industrial Robot, vol. 26, no. 5, pp. 335-341, 1999.

[10] Å. F. Berglund, F. Palmkvist, P. Nyqvist, S. Ekered, and M. Åkerman, "Evaluating Cobots for Final Assembly,” Procedia CIRP, vol. 44, pp. 175-180, 2016.

[11] O. Bonnaud and L. Fesquet, "Practice in microelectronics education as a mandatory complement to the future numeric-based pedagogy: a strategy of the French national network," International KES Conference on Smart Education and Smart E-Learning, Springer, Cham, pp. 1-8, May 2016.

[12] M. McKellop, "The future of the semiconductor industry is the Internet of Things," The Burn-In, https://www.theburnin.com/industry/semiconductors-internet-of-things/, November 28, 2018.

[13] R. R. Tummala and M. Swaminathan, System on Package, Miniaturization of the Entire System, McGraw-Hill Education, The1st edition, May 2008.

[14] T. Simonite, "Moore's Law Is Dead. Now What?,” MIT Technology Review, https://www.technologyreview.com/s/601441/moores-law-is-dead-now-what/, May 13, 2016. 
[15] O. Bonnaud, “The Multidisciplinary Approach: A Common Trend For ULSI and Thin Film Technology,” ECS Transaction, vol. 67, no. 1, pp. 147-158, 2015.

[16] Technopedia, "Internet of Everything (IoE)," https://www.techopedia.com/definition/30121/internet-of-everything-ioe, 2019.

[17] IDC, "What the IoT market has in store for Europe in 2019," https://uk.idc.com/trends/predictions, January 30,2019

[18] Enerdata, "Global Energy Statistical Yearbook 2019," https://yearbook.enerdata.net/total-energy/world-energy-production.html, March 2019.

[19] International Energy Agency, "World Energy Outlook 2019," https://www.iea.org, January 2019.

[20] Enerdata, "World Energy Production," https://yearbook.enerdata.net/total-energy/world-energy-production.html, 2019.

[21] CNFM, "Coordination Nationale pour la formation en Microélectronique et nanotechnologies," www.cnfm.fr; GIP-CNFM, March 2019.

[22] O. Bonnaud, P. Gentil, A. Bsiesy, S. Retailleau, E. D. Gergam, and J. M. Dorkel,“GIP-CNFM: a French education network moving from microelectronics to nanotechnologies," Proceeding of IEEE Global Engineering Education Conference; Amman, Jordan, pp. 122-127, April 2011.

[23] ACSIEL Alliance Électronique, "Components and systems alliance for electronics industry in France," http://www.acsiel.fr/en-GB/index.aspx, 2019.

[24] O. Bonnaud, "Practice-oriented pedagogical strategy of the French microelectronics and nanotechnologies network," 14th International Conference on Education and educational Technology, Kuala-Lumpur, Malaysia, pp. 11-19, April 2015.

[25] A. Fox, "From MOOCs to SPOCs," Communications of the ACM, vol. 56, no. 12, pp. 38-40, 2013.

[26] O. Bonnaud and L. Fesquet, "MOOC and the Practice in electrical and information engineering: complementary approaches," 15th International Conference on Information Technology Based Higher Education and Training, Istanbul, Turkey, September 2016.

[27] O. Bonnaud, "Knowledge and know-how in microelectronics; strategy of innovative practice to balance the new on-line courses approach," International Conference on Advanced Technology Innovation, Koh Samui, Thailand, pp. 10-11, June 2017.

[28] FINMINA, "Formations Innovantes en Microélectronique et Nanotechnologies," http://www.cnfm.fr/?q=fr/pr\%C3\%A9sentation_FINMINA, Innovative Training for Microelectronics and Nanotechnologies March 2019.

[29] O. Bonnaud, L. Fesquet, P. Nouet, and T. M. Brahim, "FINMINA: a French national project to promote Innovation in Higher Education in Microelectronics and Nanotechnologies," International Conference on Information Technology Based Higher Education and Training, York, UK, Proceeding of ITHET 2014, September 11-13, 2014.

[30] O. Bonnaud, "New Vision in Microelectronics Education: Smart e-Learning and Know-how, a Complementary Approach," International KES Conference on Smart Education and Smart E-Learning, pp. 267-275, 2019.

[31] F. Bruguier, P. Benoit, L. Dalmasso, B. Pradarelli, and L. Torres, “AMUSE: un escape game pour l'enseignement de la sécurité numérique,”, Proceeding of 15th JPCNFM 2018, Saint-Malo, France, vol.15, November 2018. (French)

[32] O. Bonnaud, Y. Danto, Y.Kuang,and Y. Li, "International flipped class for Chinese Honors Bachelor students in the frame of multidisciplinary fields: reliability and microelectronics," Advanced In Technology Innovation, vol. 3, no. 3, pp. 126-132, 2018.

[33] O. Bonnaud and L. Fesquet, "Innovation for education on Internet of things," Proceeding of Engineering and Technology Innovation, vol. 9, pp. 1-8, 2018.

[34] O. Bonnaud and L. Fesquet, "Communicating and smart objects: multidisciplinary topics for the innovative education in microelectronics and its applications," Proceeding of 15th International Conference Information Technology Based Higher Education and Training, IEEE Press, pp. 1-5, August 2015.

[35] O. Bonnaud, "Mandatory Matching Between Microelectronics Industry and Higher Education in Engineering Toward a Digital Society," Smart Education and e-Learning 2019, Springer, Singapore, vol. 24, pp. 255-266, 2020.

Copyright $\odot$ by the authors. Licensee TAETI, Taiwan. This article is an open access article distributed under the terms and conditions of the Creative Commons Attribution (CC BY-NC) license (https://creativecommons.org/licenses/by-nc/4.0/). 